\title{
Bloqueio Extraconal para Facectomia com Implante de Lente Intra-Ocular: Influência da Via de Acesso (superior ou inferior) na Qualidade da Anestesia *
}

\section{Extraconal Block for Cataract Extraction Surgery with Implantation of Intraocular Lens: Influence of Access Way (upper or lower) in Anesthetic Outcome}

\author{
Daniel Espada Lahoz ${ }^{1}$; Eloisa Bonetti Espada ${ }^{2}$; José Carlos Almeida Carvalho, TSA ${ }^{3}$
}

\section{RESUMO}

Lahoz DE, Espada EB, Carvalho JCA - Bloqueio Extraconal para Facectomia com Implante de Lente Intra-Ocular: Influência da Via de Acesso (superior ou inferior) na Qualidade da Anestesia

JUSTIFICATIVA E OBJETIVOS: Nas intervenções cirúrgicas para oftalmologia não se encontrou técnica de anestesia totalmente segura, a introdução da anestesia extraconal fez aumentar as indicações de cirurgias oculares com bloqueio, já que a incidência de complicações graves é menor, fato já descrito por Hay, em 1991. Os bloqueios extraconais podem ser realizados por várias vias de acesso, entre elas a superior e a inferior. O objetivo deste estudo foi avaliar qual via de acesso (superior ou inferior) promove bloqueio anestésico de melhor qualidade.

MÉTODO: Foram incluídos neste estudo 164 pacientes, de ambos os sexos, com idades entre 23 e 92 anos, estado físico ASA I a IV, índice cardíaco 1 e 2 de Goldman, com indicação de facectomia com implante de lente intra-ocular. Os pacientes foram distribuídos aleatoriamente em dois grupos de 82, de acordo com a via de acesso primária do bloqueio extraconal: grupo ES (extraconal superior) e El (extraconal inferior). A qualidade do bloqueio foi avaliada pelo aparecimento da dor no per-operatório, manutenção de movimentação das pálpebras ou do globo ocular, persistência do reflexo de Bell, número de bloqueios realizados para a obtenção de condições cirúrgicas e avaliação do bloqueio pelo cirurgião.

RESULTADOS: A via de acesso superior apresentou maior incidência de acinesia de pálpebras (via superior - $56,1 \%$; via

* Recebido do (Received from) Hospital Santa Cruz, Hospital Universitário da Universidade de São Paulo (HU-USP), São Paulo, SP

1. Mestre em Medicina na Área de Concentração de Anestesiologia pela FMUSP; Coordenador do Serviço de Anestesia em Oftalmologia da Disciplina de Anestesiologia, Dor e Terapia Intensiva da UNIFESP; Médico Assistente da Equipe de Anestesiologia da Disciplina de Anestesiologia do HC-FMUSP; Anestesiologista do Serviço de Oxigenioterapia, Anestesiologia e Inaloterapia (SOANIL) do Hospital Santa Cruz

2. Doutora em Medicina na Área de Concentração de Anestesiologia pela FMUSP; Diretora do Serviço de Anestesiologia do HU-USP; Médica Assistente da Equipe de Controle de Dor da Disciplina de Anestesiologia da FMUSP;Professora Assistente da Disciplina de Terapêutica da Clínica da UNISA

3. Livre Docente pela Disciplina de Anestesiologia da FMUSP, Diretor Clinico da Pró-Mater de São Paulo

Endereço para Correspondência (Correspondence to)

Dr. Daniel Espada Lahoz

R. Dr. Nicolau de Souza Queiroz, 297/121 Aclimação

04105-002 São Paulo, SP

E-mail: espada@ajato.com.br

Apresentado (Submitted) em 05 de setembro de 2002

Aceito (Accepted) para publicação em 07 de janeiro de 2003

(C) Sociedade Brasileira de Anestesiologia, 2003 inferior - 36,6\%) do músculo reto superior (via superior - 93,9\%: via inferior - 65,9\%), assim como menor necessidade de bloqueios complementares (via superior - 29,3\%; via inferior $42,7 \%$ ). A via de acesso inferior apresentou maior acinesia do músculo reto inferior (via superior - 72\%; via inferior - 84,1\%) sem diferença estatística.

CONCLUSÕES: Nas condições deste estudo a via extraconal superior demonstrou ser superior em relação à via extraconal inferior, como via de acesso primária para bloqueio locorregional para cirurgia de facectomia com implante de lente intra-ocular.

Unitermos: ANESTÉSICOS, Local: bupivacaína, CIRURGIA, Oftalmológica: catarata; TÉCNICAS ANESTÉSICAS, Regional: bloqueio extraconal

\section{SUMMARY}

Lahoz DE, Espada EB, Carvalho JCA - Extraconal Block for Cataract Extraction Surgery with Implantation of Intraocular Lens: Influence of Access Way (upper or lower) in Anesthetic Outcome

BACKGROUND AND OBJECTIVES: There is no completely safe anesthetic technique for ophthalmic surgery. The introduction of extraconal anesthesia has increased the number of ophthalmic surgeries with blockade since the incidence of severe complications is lower, as reported by Hay in 1991. Extraconal blockades may be induced by several access ways, among them upper and lower ways. This study aimed at evaluating the influence access ways (upper or lower) in anesthetic outcome. METHODS: Participated in this study 164 patients of both genders, aged 23 to 92 years, physical status ASA I to IV, 1 and 2 Goldman's cardiac risk index, undergoing elective cataract extraction surgery with intraocular lens implantation. Patients were randomly distributed in two groups of 82 according to primary extraconal block access way: group UE (upper extraconal), group LE (lower extraconal). Blockade quality was evaluated by the following parameters: intraoperative pain, eyelid and/or eyeball movement, persistence of Bell's reflex, number of blocks needed for eye akinesia, and surgeon's evaluation.

RESULTS: Upper extraconal approach was associated to more effective eyelid (upper access - 56.1\%; lower access $36.6 \%$ ) and superior rectus muscle akinesia (upper access - 93.9\%; lower access $65.9 \%$ ) and also a lower incidence of supplementary blocks (upper access - 29.3\%; lower access $42.7 \%$ ). The lower extraconal approach was associated to more effective inferior rectus muscle akinesia (upper access - $72 \%$; lower access - 84.1\%), however without statistical differences.

CONCLUSIONS: In the conditions of this study the upper extraconal approach was better as compared to the lower approach as the primary access way for anesthetic block for cataract extraction with intraocular lens implantation.

Key Words: ANESTHETICS, Local: bupivacaine; ANESTHETIC TECHNIQUES, Regional: extraconal block; SURGERY, Ophthalmic: cataract 


\section{INTRODUÇÃO}

A facectomia com implante de lente intra-ocular é o procedimento cirúrgico mais freqüente na população geriátrica. Muitos dos pacientes que necessitam de cirurgia oftalmológica possuem doenças associadas, beneficiando-se das vantagens da anestesia locorregional, pois a maioria das cirurgias oculares pode ser realizada sob esta técnica anestésica com ou sem sedação ${ }^{1}$.

A anestesia retrobulbar, uma das técnicas mais consagradas de bloqueio oftalmológico, consiste na injeção da solução anestésica local no interior do cone muscular formado pelos músculos próprios do bulbo ocular ${ }^{2-4}$. Embora não se tenha encontrado técnica de anestesia totalmente segura, a introdução da anestesia peribulbar fez aumentar as indicações de cirurgias oculares com bloqueio, já que a incidência de complicações graves é menor ${ }^{5}$, fato já descrito por Hay e col. (1991) ${ }^{6}$. Na anestesia peribulbar, a injeção da solução anestésica ocorre na maioria das vezes no espaço periconal ${ }^{7}$, sendo então a denominação de bloqueio peribulbar errôneo a estes. Entendemos que é preferível a utilização do termo bloqueio extraconal para os bloqueios realizados fora do cone muscular e intraconal para os bloqueios realizados dentro do cone.

O objetivo deste estudo foi comparar qual via de acesso (superior ou inferior) do bloqueio extraconal promove bloqueio anestésico de melhor qualidade.

\section{MÉTODO}

Após aprovação das Comissões de Ética em Pesquisa dos Hospitais e com o consentimento pós-informado, foram incluídos neste estudo 164 pacientes de ambos os sexos, com idades entre 23 e 92 anos, estado físico ASA I a IV, índice de risco cardíaco de Goldman de 1 e 2, com indicação cirúrgica de facectomia extracapsular com implante de lente intra-ocular. Todos os bloqueios foram realizados pelo mesmo anestesiologista.

Os pacientes foram monitorizados com cardioscopia, pressão arterial não invasiva e oximetria de pulso, após a qual realizou-se venóclise periférica em membro superior com cateter 20 ou 22G, iniciando-se hidratação pela infusão de solução de Ringer com lactato na velocidade de $3 \mathrm{ml} \cdot \mathrm{min}^{-1}$.

Os pacientes foram sedados com $50 \mu \mathrm{g}$ de fentanil, por via venosa, e doses tituladas de propofol a $0,5 \%$ até a perda do reflexo ciliar, quando era realizado o bloqueio extraconal. Quando desperto da sedação, era submetido à avaliação quanto à acinesia e à analgesia.

A acinesia foi constatada por meio da imobilidade do bulbo ocular nos quatro quadrantes, e a incapacidade de fechar com vigor a pálpebra. Foi monitorizada a analgesia pela capacidade do paciente suportar o toque corneano com a lateral de uma agulha.

Os pacientes foram agrupados segundo a via de acesso primária do bloqueio em Extraconal Superior (ES - 82 pacientes) e Extraconal Inferior (EI - 82 pacientes).
A via de acesso extraconal superior teve como referência para punção 1 a $2 \mathrm{~mm}$ da borda lateral da chanfradura supra-orbitária, com o direcionamento da agulha para a órbita até que a agulha transfixasse a pele e o tecido celular subcutâneo. Reposicionava-se a ponta da agulha em direção à fissura orbitária superior, aproximando-a ao máximo das estruturas ósseas. Após a aproximação da fissura, que ocorreu entre 30 e $35 \mathrm{~mm}$, retrocedeu-se a agulha por aproximadamente 1 a $2 \mathrm{~mm}$, sendo injetada a solução anestésica.

A via de acesso extraconal inferior teve como local de punção a junção do terço lateral com o terço medial da borda orbitária inferior. Direcionou-se a ponta da agulha para a fissura orbitária inferior, sendo introduzida de maneira paralela ao assoalho da órbita por aproximadamente $30 \mathrm{~mm}$, onde se depositou a solução anestésica.

Utilizou-se como solução anestésica bupivacaína a 0,75\% com hialuronidase (100 UTR. $\mathrm{ml}^{-1}$ ), no volume total de $7 \mathrm{ml}$. A hialuronidase foi diluída com a própria bupivacaína na proporção de 2.000 UTR para cada $20 \mathrm{ml}$ de bupivacaína a $0,75 \%$. Utilizou-se agulha hipodérmica descartável calibre 7 com $40 \mathrm{~mm}$ de comprimento.

Quando houve falha do bloqueio proposto, associou-se bloqueio complementar. Ocorrendo falha do bloqueio extraconal superior, complementava-se com o bloqueio extraconal inferior e vice-versa. A solução anestésica utilizada foi bupivacaína a $0,75 \%$ com hialuronidase (100 UTR. $\mathrm{ml}^{-1}$ ), no volume total de $3 \mathrm{ml}$. Na persistência da falha do bloqueio complementar, procedia-se ao terceiro bloqueio, intraconal ou retrobulbar, com bupivacaína a $0,75 \%$ com hialuronidase (100 UTR. $\mathrm{ml}^{-1}$ ), no volume total de $3 \mathrm{ml}$.

Para o bloqueio intraconal utilizou-se como via de acesso a junção do terço lateral com o terço medial da borda orbitária inferior. Após a ponta da agulha ter ultrapassado o equador do bulbo ocular, esta foi reposicionada súpero-medialmente em direção à junção do terço medial com o terço lateral da borda orbitária superior do olho contralateral. Este procedimento ocorreu até que houvesse a sensação da perfuração de uma folha de papel. Neste local foi administrada a solução anestésica.

Nos casos em que a falha era apenas motora palpebral, complementava-se com o bloqueio modificado de van Lint, utilizando-se apenas $3 \mathrm{ml}$ de bupivacaína a 0,75\%.

O bloqueio de van Lint modificado foi realizado injetando-se a solução anestésica aproximadamente $1 \mathrm{~cm}$ da borda lateral orbital no subcutâneo, diretamente sobre o periósteo, com direcionamento da agulha caudal e posteriormente cefálica com injeção de $1,5 \mathrm{ml}$ em cada direção.

Em seqüência à injeção do anestésico local, em qualquer fase, era realizada pressão digital firme sobre o bulbo ocular por aproximadamente 2 a 3 minutos, após a qual foi colocado um peso de $650 \mathrm{~g}$ no olho a ser operado com a finalidade de diminuir a pressão intraocular e facilitar a dispersão do anestésico local.

Com intenção de avaliar a qualidade do bloqueio, foram estudados os seguintes parâmetros: aparecimento de dor per-operatória, a manutenção da mobilidade das pálpebras, movimentação do globo (considerando-se os músculos ex- 
tra-oculares associados e em separado), persistência do reflexo de Bell, número de bloqueios intracavitários complementares necessários para se obter condições cirúrgicas e a avaliação do bloqueio pelo cirurgião, o qual não estava ciente nem da solução injetada nem da via do bloqueio utilizado, de acordo com a seguinte escala:

1) Anestesia completa, paralisia ocular total, paciente colaborativo;

2) Anestesia completa, paralisia ocular incompleta, paciente colaborativo;

3) Anestesia incompleta, paralisia ocular total, paciente com desconforto cirúrgico;

4) Anestesia incompleta, paralisia ocular incompleta, paciente com desconforto.

Para a análise estatística utilizou-se o teste do Qui-quadrado com o objetivo de verificar diferenças na distribuição de um atributo categorizado em função de outro também categorizado. Em alguns casos, o teste não pôde ser aplicado em função da baixa freqüência observada em alguns atributos. Quando o total de casos ultrapassou 40, foi utilizado o teste Qui-quadrado com correção de Yates, assim como nos casos entre 20 e 40, sendo que nenhum atributo apresentou freqüência menor que 5. Quando o número de casos era menor que 20 e a freqüência do atributo menor que 5 , utilizou-se o teste Exato de Fisher.

Para verificar se os grupos diferiam quanto à idade, peso, altura e tempo em minutos do início do bloqueio até o aparecimento de dor no período pós-operatório, utilizou-se a Análise de Variância (ANOVA).

Em todos os testes estatísticos, utilizou-se nível de significância de $5 \%$, sendo os testes realizados com auxílio do software estatístico SPSS para Windows versão 6.0.

\section{RESULTADOS}

Os dois grupos foram homogêneos quanto aos dados antropométricos, olho operado, estado físico e quanto ao índice de risco cardíaco de Goldman (Tabela I).

Na população estudada, houve predominância do estado físico ASA II e índice de risco cardíaco 1.
Não se observou diferença estatística quanto à presença de dor nos diferentes momentos estudados nos dois grupos, destacando-se a baixa incidência em todos os momentos, sendo um pouco mais elevada por ocasião da injeção subconjuntival de gentamicina (Tabela II).

Tabela I - Dados Antropométricos, Olho Operado, Estado Físico e Índice de Risco Cardíaco de Goldman dos Pacientes

\begin{tabular}{llcc}
\hline & & $\begin{array}{c}\text { Grupo ES } \\
(\mathrm{n}=82)\end{array}$ & $\begin{array}{c}\text { Grupo EI } \\
(\mathrm{n}=82)\end{array}$ \\
\hline Idade (anos) & & $70,5 \pm 11,2$ & $67,6 \pm 11,9$ \\
Peso $(\mathrm{kg})^{*}$ & & $67,3 \pm 14,8$ & $65,7 \pm 14,2$ \\
Altura $(\mathrm{cm})^{*}$ & & $163,4 \pm 11,3$ & $162,5 \pm 10,8$ \\
Sexo (\%) & Masculino & 57,3 & 47,6 \\
& Feminino & 42,7 & 52,4 \\
Olho operado (\%) & Direito & 45,1 & 53,7 \\
& Esquerdo & 54,9 & 46,3 \\
Estado físico (\%) & ASA I & 22 & 29,3 \\
& ASA II & 68,3 & 61 \\
& ASA III & 7,3 & 9,8 \\
& ASA IV & 2,4 & 0 \\
Goldman (\%) & 1 & 85,4 & 90,2 \\
& 2 & 14,6 & 9,8 \\
\hline
\end{tabular}

* Valores expressos pela Média \pm DP, demais dados em porcentagem

Tabela II - Distribuição dos Pacientes quanto o Momento de Aparecimento de Dor Per-Operatória

\begin{tabular}{lccc}
\hline Grupos & \multicolumn{3}{c}{ Surgimento da Dor } \\
\hline & $\begin{array}{c}\text { Abertura de } \\
\text { Esclera } \\
(\%)\end{array}$ & $\begin{array}{c}\text { Último Ponto de } \\
\text { Sutura da Esclera } \\
(\%)\end{array}$ & $\begin{array}{c}\text { Injeção Subconjuntival } \\
\text { de Gentamicina } \\
(\%)\end{array}$ \\
\hline ES $(n=82)$ & 1,2 & 1,2 & 17,1 \\
El $(n=82)$ & 0 & 1,2 & 13,4 \\
\hline
\end{tabular}

Após o primeiro bloqueio extraconal, não se observou diferença estatística entre os dois grupos quanto à movimentação dos músculos reto inferior, reto lateral, oblíquo inferior, oblíquo superior e do reflexo de Bell; o grupo extraconal inferior apresentou maior incidência, estatisticamente significante, da movimentação da pálpebra e do músculo reto superior em relação ao extraconal superior (Tabela III).

Tabela III - Persistência de Movimentação da Pálpebra e do Bulbo Ocular, Considerando-se os Músculos Extra-Oculares Isolados e Persistência do Reflexo de Bell após os Bloqueios Extraconais

\begin{tabular}{|c|c|c|c|c|c|c|c|c|}
\hline & \multirow[t]{2}{*}{ Pálpebras (\%) } & \multicolumn{6}{|c|}{ Músculos do Bulbo Ocular (\%) } & \multirow[t]{2}{*}{ Reflexo de Bell (\%) } \\
\hline & & RS & $\mathrm{RI}$ & $\mathrm{RL}$ & $\mathrm{RM}$ & OS & $\mathrm{OI}$ & \\
\hline \multicolumn{9}{|c|}{$1^{\circ}$ Bloqueio $(n=164)$} \\
\hline$E S(n=82)$ & 43,9 & 6,1 & 28,0 & 20,7 & 20,7 & 6,1 & 11,0 & 7,3 \\
\hline $\mathrm{El}(\mathrm{n}=82)$ & $63,4^{*}$ & 34,1 * & 15,9 & 28,0 & 29,3 & 14,6 & 15,9 & 9,8 \\
\hline \multicolumn{9}{|c|}{$2^{\circ}$ Bloqueio $(n=74)$} \\
\hline$E S(n=36)$ & 0,0 & 0,0 & 0,0 & 0,0 & 0,0 & 0,0 & 0,0 & 0,0 \\
\hline EI $(n=38)$ & 0,0 & 5,3 & 13,2 & 15,8 & 13,2 & 2,6 & 2,6 & 0,0 \\
\hline
\end{tabular}

$\mathrm{RS}=$ músculo reto superior, $\mathrm{RI}=$ músculo reto inferior, $\mathrm{RL}=$ músculo reto lateral, $\mathrm{RM}=$ músculo reto medial, OS = músculo oblíquo superior, OI = músculo oblíquo inferior, $\mathrm{n}=$ número de doentes submetidos ao bloqueio

${ }^{*} p<0,05=$ estatisticamente significante 
Após o segundo bloqueio extraconal, devido à não movimentação no grupo extraconal superior não foi possível realizar-se testes estatísticos, mas observa-se um alto índice de falhas em relação ao músculo reto lateral seguido dos músculos reto medial, reto inferior e reto superior (Tabela III). Não se observou diferença estatisticamente significante em relação ao número de bloqueios levando-se em consideração os dois grupos e todos os bloqueios realizados. O número de bloqueios necessários para se obter condições cirúrgicas foi estatisticamente significante nos pacientes que receberam como primeira opção de bloqueio o extraconal inferior, tanto na necessidade de um segundo bloqueio (tanto intracavitário como van Lint), assim como, de um terceiro bloqueio intracavitário (Tabela IV).

A soma das porcentagens do bloqueio extracone inferior totaliza $108,4 \%$. Isto se deve ao fato de que todos os pacientes submetidos a um terceiro bloqueio intracavitário receberam primeiramente o segundo bloqueio intracavitário. Os pacientes que conseguiram nesse grupo obter condições cirúrgicas com dois bloqueios intracavitários somam $34,1 \%$.

Tabela IV - Distribuição dos Pacientes, quanto aos Bloqueios Necessários para se Realizar a Cirurgia

\begin{tabular}{|c|c|c|c|c|c|}
\hline Grupos & Bloquei & acavitá & $(\%)$ & van Lint & $2^{\circ}$ Bloqueio \\
\hline & 1 & 2 & 3 & & \\
\hline$E S(n=82)$ & 70,7 & 29,3 & 0,0 & 14,6 & 43,9 \\
\hline $\mathrm{EI}(\mathrm{n}=82)$ & 57,3 & $42,6^{*}$ & $8,5^{*}$ & 16,3 & 61,0 * \\
\hline Total $(n=164)$ & 64 & 31,7 & 4,2 & 15,4 & 52,4 \\
\hline
\end{tabular}

1 = necessitou de 1 bloqueio intracavitário, 2 = necessitou de 2 bloqueios intracavitários, 3 = necessitou de 3 bloqueios intracavitários, van Lint = necessitou do bloqueio de van Lint, $2^{\circ}$ Bloqueio $=$ necessitou de um segundo bloqueio (tanto intracavitário como van Lint) e Total = número total de bloqueios necessários para adequação dos pacientes para a cirurgia proposta

${ }^{\star} p<0,05=$ estatisticamente significante

Não houve indícios de diferença estatística entre os dois grupos quanto à avaliação do bloqueio pelos cirurgiões (Tabela V).

Tabela V - Distribuição dos Pacientes quanto à Avaliação do Bloqueio pelo Cirurgião

\begin{tabular}{lcccc}
\hline Grupos & \multicolumn{5}{c}{ Avaliação do Cirurgião (\%) } \\
\hline ES $(n=82)$ & 93,9 & 6,1 & 3 & 4 \\
El $(n=82)$ & 89,0 & 7,3 & 3,7 & 0,0 \\
\hline
\end{tabular}

1 = Anestesia completa, paralisia ocular total, paciente colaborativo, 2 = Anestesia completa, paralisia ocular incompleta, paciente colaborativo, $3=$ Anestesia incompleta, paralisia ocular total, paciente com desconforto cirúrgico e 4 = Anestesia incompleta, paralisia ocular incompleta, paciente com desconforto

\section{DISCUSSÃO}

Os dados antropométricos, assim como o olho operado estão distribuídos homogeneamente entre os dois grupos. Com isso pode-se garantir que possíveis diferenças nos resultados não sejam decorrentes destes.

Como a doença ocular em questão, catarata, não põe a vida do doente em risco, assim como não se trata de urgência ou emergência, normalmente há tempo hábil para compensar as doenças associadas, justificando assim as boas condições em que estes doentes freqüentemente se encontram na sala cirúrgica, o que pode ser visto pela classificação do estado físico (ASA) e pelo índice de risco cardíaco de Goldman.

Não foi encontrada diferença estatisticamente significante entre os dois grupos estudados quanto à dor per-operatória. A queixa de dor ocorreu com maior freqüência no momento da injeção subconjuntival de gentamicina, ressaltando a importância desse momento cirúrgico. Não foram encontrados relatos na literatura sobre tal fato.

A tomografia computadorizada, logo após a realização dos bloqueios tanto intraconal como dos extraconais, demonstra que a solução anestésica expande-se livremente entre os espaços intra e extra-cone, independentemente do local em que foi injetada ${ }^{8}$. A existência de falha de difusão da solução anestésica, talvez pelo fato do volume anestésico não ter sido suficiente, explicaria a diferença estatisticamente significativa quanto à movimentação das pálpebras e do músculo reto superior nos bloqueio extraconal inferior. Estes dados coincidem parcialmente com os encontrados na literatura ${ }^{7}$. Os bloqueios em que se utiliza a via de acesso inferior, há faIha nos músculos reto medial e do reto superior.

Observando-se os pacientes que obtiveram anestesia satisfatória com dois bloqueios intracavitários, não foi encontrada diferença estatisticamente significante. Considerando-se os pacientes que necessitaram de mais de um bloqueio intracavitário (somando-se o $2^{\circ} \mathrm{com}$ o $3^{\circ}$ bloqueio intracavitário), constatou-se que o grupo extraconal inferior apresentou maior necessidade de bloqueio complementar (42,6\%). Evidenciou-se também que o grupo extraconal inferior apresentou maior necessidade de um segundo bloqueio (61\%), indiferentemente do bloqueio utilizado ser o van Lint ou intracavitário.

Quanto à necessidade de um terceiro bloqueio intracavitário (bloqueio intraconal) nos pacientes que receberam o segundo bloqueio intracavitário, observou-se que apenas o grupo extraconal inferior apresentou necessidade de um terceiro bloqueio. Verificou-se que após a realização do segundo bloqueio intracavitário, não houve a necessidade da realização do bloqueio de van Lint, provavelmente pelo extravasamento da solução anestésica, bloqueando a motricidade do músculo orbicular do olho.

A avaliação da técnica anestésica e conforto cirúrgico pelo cirurgião não apresentou diferença estatisticamente significava entre os dois grupos, nos quais $91,5 \%$ receberam nota I (anestesia completa, paralisia ocular total, doente colaborativo). Campos e col. ${ }^{9}$ relataram que $98 \%$ dos bloqueios foram classificados como ótimos pelos cirurgiões.

Katayama e col. ${ }^{10}$ citaram que a técnica peribulbar com a injeção em apenas um local por meio de agulha de pequeno calibre apresenta alta incidência de falhas, optando por esse motivo pelo bloqueio na dupla punção. No entanto, não apresentam os valores obtidos nos resultados.

Ahmad e col. ${ }^{1}$ relataram a necessidade de rebloqueios intracavitários de $3 \%$ por falha na acinesia do olho, valendo lem- 
brar que estes autores realizam técnica por duas punções, uma superior e outra inferior. Nessa situação, em nosso trabalho encontramos $4,3 \%$ de pacientes que necessitaram do terceiro bloqueio.

Os mesmos autores relatam que nesses bloqueios o músculo reto medial é o que apresenta maior número de falhas de acinesia. No presente estudo foi encontrado maior índice de falhas do músculo reto lateral, seguido da falha do reto medial e inferior.

Observou-se neste estudo que $64 \%$ dos pacientes necessitaram de apenas uma punção para o bloqueio (por qualquer que a primeira via), não justificando a dupla punção como primeira alternativa, visto que para se avaliar a necessidade ou não da segunda punção, haveria um aumento de apenas 15 minutos para o início da cirurgia.

Nas condições deste estudo a via extraconal superior demonstrou ser melhor em relação à via extraconal inferior, como via de acesso primária para bloqueio locorregional para cirurgia de facectomia com implante de lente intra-ocular.

\section{Extraconal Block for Cataract Extraction Surgery with Implantation of Intraocular Lens: Influence of Access Way (upper or lower) in Anesthetic Outcome}

Daniel Espada Lahoz, M.D., Eloisa Bonetti Espada, M.D., José Carlos Almeida Carvalho, TSA, M.D.

\section{INTRODUCTION}

Facectomy with intraocular lens implantation is the most frequent surgical procedure among elderly patients. Several patients needing ophthalmic surgeries have associated diseases and benefits from loco-regional anesthesia since most ophthalmic surgeries may be performed under this technique, with or without sedation ${ }^{1}$.

Retrobulbar anesthesia, one of the most popular ophthalmic block techniques, consists on the injection of local anesthetic solution inside the muscle cone formed by the muscles of the bulb of eye themselves ${ }^{2-4}$. Although the lack of a completely safe anesthetic technique, peribulbar anesthesia has increased the number of ophthalmic surgeries with blockade since there is a low incidence of severe complications ${ }^{5}$ as described by Hay et al. $(1991)^{6}$. Most of the times, peribulbar anesthesia is induced in the periconal space ${ }^{7}$ and, in these cases, the name peribulbar block is misleading. We prefer the term extraconal block for blockades performed outside the muscle cone and intraconal block for blockades performed inside the cone.

This study aimed at comparing extraconal block access ways (upper or lower) in terms of better anesthetic outcome.

\section{METHODS}

After the Hospitals' Ethics Committee approval and their informed consent, participated in this study 164 patients of both genders, aged 23 to 92 years, physical status ASA I to IV, 1 and 2 Goldman's cardiac risk index, scheduled for extracapsular facectomy with intraocular lens implantation. The same anesthesiologist induced all blockades.

Patients were monitored with cardioscopy, non-invasive blood pressure and pulse oximetry. Peripheral venoclysis was performed in an upper limb with 20 or $22 \mathrm{G}$ catheters and hydration was started with lactated Ringer's solution at a rate of $3 \mathrm{ml}$. $\mathrm{min}^{-1}$.

Patients were sedated with $50 \mu \mathrm{g}$ intravenous fentanyl and $0.5 \%$ propofol titrated doses until loss of ciliary reflex, when extraconal blockade was induced. At sedation emergence, patients were submitted to evaluation of akinesia and analgesia.

Akinesia was confirmed by eyeball immobility in the four quadrants and the inability to strongly close eyelids. Analgesia was monitored by the ability of standing corneal touching with the side of a needle.

Patients were grouped according to primary blockade access way in Upper Extraconal (UE - 82 patients) and Lower Extraconal (LE - 82 patients).

Reference for upper extraconal puncture was 1 to $2 \mathrm{~mm}$ from the lateral border of the supra-orbital notch, directing the needle toward the orbit until crossing skin and subcutaneous tissue. Needle was then redirected toward the upper orbital fissure getting as close as possible to bone structures. After fissure approximation, between 30 and $35 \mathrm{~mm}$, needle was removed approximately 1 to $2 \mathrm{~mm}$ and anesthetic solution was injected.

Reference for lower extraconal puncture was the junction of the lateral third with the medial third of the lower orbital border. Needle was directed to the lower orbital fissure and introduced parallel to the orbit floor for approximately $30 \mathrm{~mm}$ when anesthetic solution was injected.

Anesthetic solution was $0.75 \%$ bupivacaine with hyaluronidase (100 UTR. ml ${ }^{-1}$ ) in a total volume of $7 \mathrm{ml}$. Hyaluronidase was diluted in bupivacaine in a ratio of 2000 UTR for each $20 \mathrm{ml}$ of $0.75 \%$ bupivacaine. Disposable $21 \mathrm{G}$ $40 \mathrm{~mm}$ length needle was used.

Supplementary block was induced in case of failure of proposed blockade. When upper extraconal block failed, it was supplemented with lower extraconal block and vice versa. Anesthetic solution was $0.75 \%$ bupivacaine with hyaluronidase (100 UTR. $\mathrm{ml}^{-1}$ ) in a total volume of $3 \mathrm{ml}$. In case of supplementary block failure, a third intraconal or extrabulbar blockade was induced with $0.75 \%$ bupivacaine with hyaluronidase (100 UTR. $\mathrm{ml}^{-1}$ ) in a total volume of $3 \mathrm{ml}$. Intraconal block access way was the junction of the lateral third with the medial third of the lower orbital border. After overcoming eyeball ecuador, needle was supero-medially repositioned toward the junction of the medial third and the lateral third of the upper orbital border of the opposite eye. 
Procedure continued until the sensation of perforating a paper sheet, when anesthetic solution was injected.

If there was only eyelid motor failure, it was supplemented by van Lint modified blockade with just $3 \mathrm{ml}$ of $0.75 \%$ bupivacaine.

Modified van Lint blockade was induced by injecting the anesthetic solution at approximately $1 \mathrm{~cm}$ of the subcutaneous orbital lateral border, directly over the periostium, directing the needle caudal and then cephalad and injecting $1.5 \mathrm{ml}$ in each direction.

Following local anesthetic injection, in any stage, firm digital pressure was applied on the eyeball for approximately 2 to 3 minutes and a $650 \mathrm{~g}$ weigh was placed on the eye to be operated on, aiming at decreasing intraocular pressure and help local anesthetic spread.

The following parameters were studied to evaluate blockade quality: perioperative pain, eyelid mobility, eyeball movements (considering extra-ocular muscles associated and in separate), Bell's reflex persistence, number of supplementary intracavitary blocks needed for surgical conditions and blockade evaluation by the surgeon who was blind to the injected solution and access way, according to the following scale:

1) Total anesthesia, total eye paralysis, cooperative patient;

2) Total anesthesia, partial eye paralysis, cooperative patient;

3) Partial anesthesia, total eye paralysis, patient with surgical discomfort;

4) Partial anesthesia, partial eye paralysis, patient with discomfort.

Chi-square test was applied to check differences in the distribution of a categorized attribute as a function of another attribute also categorized. In some cases the test could not be applied as a function of the low frequency of some attributes. When the total number of cases was above 40 , Chi-square test with Yates correction was applied, as well in cases between 20 and 40, and no attribute had a frequency below 5. Fisher's Exact test was applied when the number of cases was below 20 and attributes frequency was below 5 .

Analysis of Variance (ANOVA) was applied to check differences in age, weight, height and time in minutes from beginning of blockade to postoperative pain complaint.

For all tests, significance level was $5 \%$ and tests were performed with the aid of statistical software SPPSS for Windows 6.0.

\section{RESULTS}

Both groups were homogeneous in demographics data, operated eye, physical status and Goldman's cardiac risk index (Table I).
Table I - Patients Demographics, Operated Eye, Physical Status and Goldman's Cardiac Risk Index

\begin{tabular}{llcc}
\hline & & $\begin{array}{c}\text { Group UE } \\
(\mathrm{n}=82)\end{array}$ & $\begin{array}{c}\text { Group LE } \\
(\mathrm{n}=82)\end{array}$ \\
\hline Age (years) & & $70.5 \pm 11.2$ & $67.6 \pm 11.9$ \\
Weight $(\mathrm{kg})^{*}$ & & $67.3 \pm 14.8$ & $65.7 \pm 14.2$ \\
Height $(\mathrm{cm})^{*}$ & & $163.4 \pm 11.3$ & $162.5 \pm 10.8$ \\
Gender (\%) & Male & 57.3 & 47.6 \\
& Female & 42.7 & 52.4 \\
Operated eye (\%) & Right & 45.1 & 53.7 \\
& Left & 54.9 & 46.3 \\
Physical status (\%) & ASA I & 22 & 29.3 \\
& ASA II & 68.3 & 61 \\
& ASA III & 7.3 & 9.8 \\
& ASA IV & 2.4 & 0 \\
& 1 & 85.4 & 90.2 \\
Goldman (\%) & 2 & 14.6 & 9.8 \\
\hline
\end{tabular}

*Values expressed in Mean \pm SD; other data in percentages

There has been a predominance of physical status ASAII and cardiac risk index 1 . There has been no statistical difference in pain in different studied moments between groups. There has been a low incidence of pain in all moments, being it slightly higher during sub-conjuntival gentamicin injection (Table II).

Table II - Patients Distribution as to Perioperative Pain Referral

\begin{tabular}{lccc}
\hline Groups & \multicolumn{3}{c}{ Pain Referral } \\
\hline & $\begin{array}{c}\text { Opening of } \\
\text { the Sclera (\%) }\end{array}$ & $\begin{array}{c}\text { Last Sclera } \\
\text { Suture (\%) }\end{array}$ & $\begin{array}{c}\text { Subconjuntival } \\
\text { Gentamicin Injection (\%) }\end{array}$ \\
\hline UE $(n=82)$ & 1.2 & 1.2 & 17.1 \\
LE $(n=82)$ & 0 & 1.2 & 13.4 \\
\hline
\end{tabular}

There has been no statistical difference between groups, afterfirst extraconal block, in inferior rectus, lateral rectus, inferior obliquous, superior obliquous muscles movement and Bell's reflex; lower extraconal group has shown higher and statistically significant eyelid and superior rectus muscle movement as compared to the upper extraconal group (Table III).

After the second extraconal block, it was impossible to perform statistical analysis in the upper extraconal groups due to lack of movement, but there has been a significant number of failures in lateral rectus muscle, followed by medial rectus, inferior rectus and superior rectus muscles (Table III).

There has been no statistically significant difference in the number of blockades, considering both groups and all blockades induced. The number of blockades to reach surgical conditions was statistically significant in patients receiving lower extraconal block as the first option, both in the need for a second blockade (both intracavitary and van Lint) and for a third intracavitary blockade (Table IV). 
Table III - Persistence of Eyelid and Eyeball Movements Considering Each Extraocular Muscle, and Persistence of Bell's Reflex after Extraconal Blocks

\begin{tabular}{|c|c|c|c|c|c|c|c|c|}
\hline & \multirow[t]{2}{*}{ Eyelids (\%) } & \multicolumn{6}{|c|}{ Eyeball Muscles (\%) } & \multirow[t]{2}{*}{ Bell's Reflex (\%) } \\
\hline & & SR & IR & LR & MR & so & 10 & \\
\hline \multicolumn{9}{|c|}{$1^{\text {st }}$ Blockade $(n=164)$} \\
\hline $\operatorname{LE}(\mathrm{n}=82)$ & 63.4 * & 34.1 * & 15.9 & 28.0 & 29.3 & 14.6 & 15.9 & 9.8 \\
\hline \multicolumn{9}{|c|}{$2^{\text {nd }}$ Blockade $(n=74)$} \\
\hline UE $(n=36)$ & 0.0 & 0.0 & 0.0 & 0.0 & 0.0 & 0.0 & 0.0 & 0.0 \\
\hline $\operatorname{LE}(n=38)$ & 0.0 & 5.3 & 13.2 & 15.8 & 13.2 & 2.6 & 2.6 & 0.0 \\
\hline
\end{tabular}

$\mathrm{SR}=$ superior rectus muscle, $\mathrm{IR}=$ inferior rectus muscle, $\mathrm{LR}=$ lateral rectus muscle, $\mathrm{MR}=$ medial rectus muscle, $\mathrm{SO}=\mathrm{superior}$ oblique muscle, $\mathrm{IO}=$ inferior oblique muscle, $\mathrm{n}=$ number of patients submitted to the blockade

${ }^{*} p<0.05=$ statistically significant

Table IV - Patients Distribution as to Blockades Needed to Perform Surgery

\begin{tabular}{|c|c|c|c|c|c|}
\hline \multirow[t]{2}{*}{ Groups } & \multicolumn{3}{|c|}{ Intracavitary Block (\%) } & \multirow[t]{2}{*}{ van Lint } & \multirow[t]{2}{*}{$2^{\text {nd }}$ Blockade } \\
\hline & 1 & 2 & 3 & & \\
\hline UE $(n=82)$ & 70.7 & 29.3 & 0.0 & 14.6 & 43.9 \\
\hline $\operatorname{LE}(n=82)$ & 57.3 & $42.6^{*}$ & $8.5^{*}$ & 16.3 & 61.0 * \\
\hline Total $(n=164)$ & 64 & 31.7 & 4.2 & 15.4 & 52.4 \\
\hline
\end{tabular}

1 = needed 1 intracavitary block, 2 = needed 2 intracavitary blocks, $3=$ needed 3 intracavitary blocks, van Lint $=$ needed van Lint block, $2^{\text {nd }}$ Blockade $=$ needed a second (both intracavitary and van Lint) block and Total = total number of blockades needed to perform surgery

${ }^{*} p<0.05=$ statistically significant

The sum of lower extraconal blockade percentage is equal to $108.4 \%$. This is because all patients submitted to a third intracavitary block had already received a second intracavitary block. Patients in this group reaching surgical conditions with two blockades added $34.1 \%$.

There has been no statistical difference in both groups in surgeon's evaluation of blockade (Table V).

Table $\mathrm{V}$ - Patients Distribution as to Blockade Evaluation by the Surgeon

\begin{tabular}{lcccc}
\hline Groups & \multicolumn{4}{c}{ Surgeon's Evaluation (\%) } \\
\hline UE $(n=82)$ & 1 & 2 & 3 & 4 \\
\hline LE $(n=82)$ & 83.9 & 6.1 & 0.0 & 0.0 \\
\hline
\end{tabular}

$1=$ Total anesthesia, total eye paralysis, cooperative patient, 2 = Total anesthesia, partial eye paralysis, cooperative patient, $3=$ Partial anesthesia, total eye paralysis, patient with surgical discomfort and $4=$ Partial anesthesia, partial eye paralysis, patient with discomfort

\section{DISCUSSION}

Demographics data and operated eye were homogeneous between groups and this assures that possible differences in results are not a consequence of them.

Since cataract is neither life-threatening nor urgency or emergency, in general there is enough time to compensate associate diseases, thus justifying the good conditions in which patients often are presented to the operating room and this was confirmed by physical status classification (ASA) and Goldman's cardiac risk index.
There has been no statistically significant difference between groups in perioperative pain. Pain complaint has been more frequent at subconjunctival gentamicin injection, highlighting the importance of this surgical moment. No reports were found in the literature about such fact.

CT scan soon after intraconal and extraconal blocks has shown that the anesthetic solution is freely spread between intra and extraconal spaces, regardless of the injection site ${ }^{8}$. Failure in anesthetic solution spread, probably due to insufficient anesthetic volume, would explain statistically significant differences in eyelid and superior rectus muscle movement in lower extraconal blockades. These data partially coincide with the literature ${ }^{7}$. Failure in lower access blockade was observed in medial rectus and superior rectus muscles. There has been no statistically significant difference among patients with satisfactory anesthesia with two intracavitary blocks. Considering patients needing more than one intracavitary block (adding the $2^{\text {nd }}$ and the $3^{\text {rd }}$ intracavitary block), the lower extraconal group needed more supplementary blockades $(42.6 \%)$. It has also been observed that the lower extraconal group had a higher need for a second blockade $(61 \%)$ regardless of the type of blockade - van Lint or intracavitary.

Among patients receiving the second intracavitary block, only the lower extraconal group needed a third blockade. There was no need for van Lint block after the second intracavitary block, probably due to anesthetic leakage blocking mobility orbicular muscle of eye.

There were no statistically significant differences between groups in anesthetic technique and surgical comfort evaluated by the surgeon, where $91.5 \%$ had grade I (total anesthesia, total eye paralysis, cooperative patient). Campos et al. ${ }^{9}$ have reported $98 \%$ of excellent blockades according to surgeons.

Katayama et al. ${ }^{10}$ have mentioned that the peribulbar technique with injection on a single site with fine needle has a high incidence of failures and have decided for the double puncture method. However, they have not presented values of results.

Ahmad et al. ${ }^{1}$ have reported $3 \%$ need for intracavitary reblocks due to eye akinesia failure and it should be mentioned that such authors used the double puncture method 
with an upper and a lower puncture. In this situation we have found in our study $4.3 \%$ of patients needing a third blockade. The same authors have reported that in such blockades, the medial rectus muscle has the highest incidence of akinesia failures. Our study has found a higher incidence of failures in the lateral rectus muscle, followed by medial and inferior rectus muscles.

It has been observed in this study that $64 \%$ of patients needed just one puncture for the blockade (by any access way), not justifying double puncture as the first choice, since the evaluation of the need for a second puncture or not would delay surgery beginning in just 15 minutes.

In the conditions of our study, upper extraconal access way was better as compared to the lower extraconal access way as the primary access way for loco-regional block for cataract surgery with intraocular lens implantation.

\section{REFERÊNCIAS - REFERENCES}

01. Ahmad S, Ahmad A, Benzon HT - Clinical experience with the peribulbar block for ophthalmologic surgery. Reg Anesth, 1993;18:184-188.

02. Knapp H - On cocaine and its use in ophthalmic and general surgery. Arch Ophthalmol. 1884;13:402-448.

03. Vanetti LFA - Anestesia para Oftalmologia, em: Auler Jr JOC, Vane LA - Curso de Atualização - SAESP, São Paulo, Atheneu, 1992:554-565.

04. Feitl ME, Krupin T - Anestesia retrobulbar. Clin Oftalmol Am Norte, 1990;3:83-91.

05., Freitas $\mathrm{ACH}$, Espirandelli LC - Parada respiratória após bloqueio peribulbar. Relato de caso. Rev Bras Anestesiol, 1997;47:134-137.

06. Hay A, Flynn HW, Hoffman Jl et al - Needle penetration of globe during retrobulbar and peribulbar injections. Ophthalmology, 1991;98:1017-1024.

07. Davis II DB, Mandel MR - Anestesia peribulbar. uma revisão da técnica e das complicações. Clin Oftalmol Am Norte, 1990;3: 101-110.

08 . Wong DHW - Regional anesthesia for intraocular surgery. Can J Anaesth, 1993;40:635-657.

09. Campos AR, Azevedo FJT, Silva LC - Bloqueio peribulbar: uma alternativa para cirurgia oftálmica. Rev Bras Anestesiol, 1989;39:287-291.

10. Katayama M, Zambotti HC, Vieira JL et al - Bloqueio peribulbar e retrobulbar em cirurgia oftálmica. Estudo clínico comparativo com bupivacaína a 0,75\%, com e sem hialuronidase. Rev Bras Anestesiol, 1993;43:159-166.

\section{RESUMEN}

Lahoz DE, Espada EB, Carvalho JCA - Bloqueo Extraconal para Facectomia con Implantación de Lente Intra-Ocular: Influencia de la Vía de Acceso (superior o inferior) en la calidad de la Anestesia

JUSTIFICATIVA Y OBJETIVOS: En las intervenciones quirúrgicas para oftalmología no se encontró técnica de anestesia totalmente segura, la introducción de la anestesia extraconal hizo que aumentasen las indicaciones de cirugías oculares con bloqueo, ya que la incidencia de complicaciones graves es menor, un hecho ya descrito por Hay, en 1991. Los bloqueos extraconales pueden ser realizados por varias vías de acceso, entre ellas, la superior y la inferior. El objetivo de este estudio fue evaluar cual vía de acceso (superior o inferior) promueve bloqueo anestésico de mejor calidad.

MÉTODO: Fueron incluidos en este estudio 164 pacientes, de ambos sexos, con edades entre 23 y 92 años, estado físico ASA I a IV, índice cardíaco 1 y 2 de Goldman, con indicación de facectomia con implantación de lente intra-ocular. Los pacientes fueron distribuidos aleatoriamente en dos grupos de 82 de acuerdo con la vía de acceso primaria del bloqueo extraconal: grupo ES (extraconal superior) y EI (extraconal inferior). La calidad del bloqueo fue evaluada por el aparecimiento de dolor en el per-operatorio, manutención de movimentación de las pálpebras o del globo ocular. persistencia del reflejo de Bell, número de bloqueos realizados para la obtención de condiciones quirúrgicas y evaluación del bloqueo por el cirujano.

RESULTADOS: La vía de acceso superior presentó mayor incidencia de acinesis de pálpebras (Vía superior - $56,1 \%$; vía inferior - 36,6\%) del músculo recto superior (vía superior 93,9\%; vía inferior 65,9\%), así como menor necesidad de bloqueos complementares (vía superior 29,3\%; vía inferior $42,7 \%)$. La vía de acceso inferior presentó mayor acinesis del músculo recto inferior (vía superior $72 \%$; vía inferior - $84,1 \%$ ) sin diferencia estadística.

CONCLUSIONES: En las condiciones de este estudio, la vía extraconal superior demostró que es superior en relación a la vía extraconal inferior, como vía de acceso primaria para bloqueo locorregional para cirugía de facectomia con implantación de lente intra-ocular. 